\title{
Deckungsbeitragsrechnung und ganzzahlige Programmierung
}

\author{
Dipl.-Kfm. Martin Eling, St. Gallen, und Dipl.-Kfm. Andreas Rohleder, Münster
}

\section{Ausgangssituation und Planungsvorgaben}

Die Steiger $\mathbf{G m b H}$ ist ein kleiner Hersteller von hölzernen Gartenleitern. Die letztjährige Marketing-Idee, diese Gartenleitern auch in den USA zu vertreiben, endete mit einer von der Firma Steiger jüngst verlorenen Schadenersatzklage durch einen von einer Steiger-Leiter gefallenen amerikanischen Kunden (vgl. http://www.snopes.com/radiotv/tv/qvc.asp). Der unverzüglich zu leistende Schadenersatz hat die Steiger $\mathrm{GmbH}$ in eine akute Liquiditätskrise gestürzt. Der Geschäftsführer, Herr Steiger, hat daraufhin angeordnet, alle Entscheidungen für das kommende Quartal so auszurichten, dass die zu leistenden Auszahlungen minimiert werden. Für jedes der von Firma Steiger bedienten Marktsegmente hat er für diesen Zeitraum zusätzlich einen Segmentdeckungsbeitrag vorgegeben, der nicht unterschritten werden soll. Dieser beträgt z. B. $150.000 €$ für das Marktsegment ,,private Haushalte“.

Als Controller der Firma Steiger haben Sie - wie so oft zunächst nicht über die Ideen von Herrn Steiger zu befinden, sondern müssen die optimalen operativen Einkaufs-, Produktions- und Verkaufspläne des Marktsegments „private Haushalte“ für das nächste Quartal erstellen. In diesem Marktsegment werden nur zwei Leitervarianten angeboten. „Die Große“ ist vier Meter lang und hat 14 Sprossen. „Die Kleine“ ist nur drei Meter lang und hat zehn Sprossen. Die Sprossen werden montagebereit fremdbezogen. Die Holme links und rechts müssen in einem ersten Arbeitsgang halbautomatisch aus Rohlingen, die je einen Meter lang sind, zusammengeleimt werden. Die Arbeitszeit beträgt pro Leimung zehn Sekunden. In einem zweiten Arbeitsgang werden die Leitern dann ebenfalls halbautomatisch endmontiert. Als Faustregel für die
Endmontage gilt seit vielen Jahren ,drei Sekunden pro Sprosse“. Für jede Leimung und jede Sprossen-Montage werden jeweils 20 Milliliter Leim verbraucht. Wesentliche Trocknungszeiten fallen nicht an. Eine Montagestunde wird mit $250 €$ auszahlungswirksam. Es standen im letzten Jahr 1.500 Montagestunden zur Verfügung. Die Lieferanten der Steiger $\mathrm{GmbH}$ bestehen neuerdings allesamt auf Barzahlung bei Lieferung. Ihr Angebot für das kommende Quartal kann Tab. 1 entnommen werden.

Nur mit größter Mühe (genauer gesagt, gegen Gewährung von 3\% Skonto) konnten die Absatzmittler zum Verzicht auf ihr sonst übliches Zahlungsziel überredet werden. Ihre voraussichtliche Nachfrage nach den Steiger-Produkten im kommenden Quartal kann Tab. 2 entnommen werden.

\section{Operationalisierung und Diskussion der Planungsvorgaben}

Wie können Sie die Planungsvorgaben von Herrn Steiger umsetzen? Die Vorgabe besteht darin, alle Entscheidungen im Marktsegment ,,private Haushalte“ im folgenden Quartal so auszurichten, dass die dadurch zu leistenden Auszahlungen minimiert werden. Allerdings ist neben dieser zu minimierenden Zielfunktion eine Mindestbedingung einzuhalten. Der Segmentdeckungsbeitrag soll $150.000 €$ nicht unterschreiten. Diese Forderung muss als , $\geq$ “-Nebenbedingung in der Optimalplanung berücksichtigt werden. Aus kaufmännischer Sicht ist unmittelbar ersichtlich, dass die Zielvorgabe nur unter sehr restriktiven Prämissen sinnvoll sein kann. Als solche wäre beispielsweise ein drohender Konkurs wegen Illiquidität im nächsten Quartal denkbar. Die Zahlungsunfähigkeit müsste dann zwischen

\begin{tabular}{|c|c|c|c|c|}
\hline Artikel & Lieferant & Mindestmenge & Höchstmenge & Angebotspreis \\
\hline Rohlinge & Waldmann & & $10.000[$ Stück] & $0,40[€ /$ Stück] \\
\hline Rohlinge & Holzer & & $7.000[$ Stück] & $0,35[€ /$ Stück] \\
\hline Sprossen & Waldmann & 5.000 [Stück] & $10.000[$ Stück] & $0,13[€ /$ Stück] \\
\hline Sprossen & Lindenbaum & 5.000 [Stück] & $20.000[$ Stück] & $0,15[€ /$ Stück] \\
\hline Leim & ABFS & & & $2,50[€ /$ Liter] \\
\hline
\end{tabular}

Tab. 1: Angebotsmengen und -preise

\begin{tabular}{|c|c|c|c|c|}
\hline Artikel & Kunde & Mindestmenge & Höchstmenge & Listenpreis \\
\hline „Die Große“ & Blumenland & & 800 [Stück] & 139,18 [€/Stück] \\
\hline „Die Große“ & Gartencenter & & $500[$ Stück] & 144,33 [€/Stück] \\
\hline „Die Kleine“ & Gartencenter & 1.000 [Stück] & $2.000[$ Stück] & $100,00[€ /$ Stück] \\
\hline „Die Kleine“ & Gelle-Versand & & 1.500 [Stück] & $97,94[€ /$ Stück] \\
\hline
\end{tabular}

Tab. 2: Nachfragemengen und -preise 
den Auszahlungen im Einkauf und den Einzahlungen im Verkauf eintreten. Dabei wäre anzunehmen, dass der verfügbare Kreditrahmen bereits voll ausgeschöpft ist und keine sonstigen Freiheitsgrade in der Finanzierung bestehen.

Jedoch würde die Forderung, die Auszahlungen im kommenden Quartal zu minimieren, isoliert betrachtet zu einer Einstellung der Produktion führen. Nur in diesem Fall sind keine weiteren Auszahlungen zu leisten. Der damit verbundene Verzicht auf mögliche Gewinne bzw. Deckungsbeiträge erscheint Ihnen aus kaufmännischer Sicht nicht sinnvoll. Zudem stehen weitere Planungsvorgaben einer Einstellung des Betriebs entgegen. Zum einen ist eine Mindestmenge der „Kleinen“ für das Gartencenter zu produzieren. Zum anderen soll ein Mindestdeckungsbeitrag von $150.000 €$ erzielt werden, der nur durch Fortführung der unternehmerischen Tätigkeit erwirtschaftet werden kann.

Darüber hinaus fällt Ihnen auf, dass die ausschließliche Betrachtung des Segments „private Haushalte“ gegebenenfalls bestehende Interdependenzen zu anderen Marktsegmenten zerschneidet. Als ein Beispiel wäre hier ein möglicher Wettbewerb um knappe Maschinenkapazitäten (bzw. Montagestunden) zu nennen. Auch die Beschränkung des Planungshorizonts auf ein Quartal führt kurzfristig zu Entscheidungen, die bei längerfristiger Betrachtung unter Umständen suboptimal sind. Des Weiteren erscheint Ihnen die Höhe des Segmentdeckungsbeitrags willkürlich festgelegt worden zu sein. Dabei könnte die Vorgabe eines zu hohen Segmentdeckungsbeitrags das Problem eventuell unlösbar machen.

Neben diesen grundsätzlichen Problemen erscheint auch die Informationslage unzureichend. Dabei fehlen insbesondere Informationen zu den Zahlungsströmen des kommenden Quartals. Fraglich ist zunächst zu welchen Zeitpunkten welche Zahlungen ein- bzw. ausgehen werden. Inwiefern diese Zahlungen und andere Planungsvorgaben mit Unsicherheit behaftet sind, wird ebenfalls bislang nicht problematisiert. Stattdessen werden lediglich Faustregeln im Sinne von Erwartungswerten vorgegeben. Wichtig erscheint Ihnen auch die Frage, ob die zukünftigen Zahlungen variabel oder fix, also überhaupt noch disponibel sind. Zudem könnten sonstige, bislang nicht genannte Ein- und/oder Auszahlungen, z. B. für Transport oder Versicherung, eine wichtige Rolle spielen.

Vor dem Hintergrund der angespannten Liquiditätslage sind auch weitere Informationen hinsichtlich der Finanzierungssituation der Steiger $\mathrm{GmbH}$ einzuholen. $\mathrm{Zu}-$ nächst ist zu überlegen, ob auf andere Finanzierungsarten, wie beispielsweise Lieferantenkredite, Vorauskasse der Kunden, Factoring oder Überbrückungskredite zurückgegriffen werden kann. Auch eine Beleihung vorhandener Vermögensgegenstände kommt prinzipiell in Frage. Falls tatsächlich die Zahlungsunfähigkeit droht, sollten auch die vertraglichen Konsequenzen einer Nichterfüllung von Mindestmengen (Konventionalstrafen) in die Planungsüberlegungen mit eingehen.
Schließlich fehlen Ihnen Informationen bezüglich der Marktsituation der Steiger-Leitern. Es liegen lediglich bestimmte Höchstmengen der Absatzmittler vor, jedoch keine Preis-Absatz-Funktionen oder andere Informationen zur Beurteilung des Marktpotenzials der Steiger-Produkte. Stellvertretend für weitere offene Fragen verbleibt auch noch zu klären, ob die genannten Preise brutto oder netto, also inklusive oder exklusive Umsatzsteuer zu verstehen sind.

\section{Deckungsbeitragsrechnung und die Fiktion konstanter Stückdeckungsbeiträge}

Die Liquidität ist zwar eine wichtige Voraussetzung für den Fortbestand des Unternehmens, jedoch wird bei der Auszahlungsminimierung weder eine Fixkostendeckung noch eine darüber hinausgehende Gewinnerzielung berücksichtigt. Daher wollen Sie statt der Auszahlungsminimierung eine andere Zielgröße in die Planung einbeziehen. Dabei handelt es sich um die Maximierung des Deckungsbeitrags (DB). Der Deckungsbeitrag bezeichnet den Überschuss einer Erlösgröße über diejenigen Kosten, die dieser Erlösgröße eindeutig, also ohne Schlüsselung von Fixkosten, zugeordnet werden können. Der Deckungsbeitrag wird berechnet, indem vom Nettoerlös die variablen Kosten abgezogen werden. Er zeigt damit den Beitrag der einzelnen Erlösgröße zur Deckung der Fixkosten bzw. nach Deckung der Fixkosten zum Gewinn des Unternehmens. Eine mehrstufige Deckungsbeitragsrechnung erlaubt dabei eine Profitabilitätsanalyse auf verschiedenen Hierarchieebenen wie Marktsegmenten, Kundengruppen oder Produktgruppen (vgl. dazu auch Coenenberg, 2003, S. 233 ff.). Abb. 1 zeigt eine mögliche mehrstufige Deckungsbeitragshierarchie am Beispiel der Steiger $G m b H$.

Die Deckungsbeitragshierarchie bildet die Grundlage einer differenzierten Profitabilitätsanalyse auf jeder der dargestellten Hierarchieebenen. Um beispielsweise den Deckungsbeitrag des Kunden 1 (Blumenland) zu ermitteln, müssen Sie den Erlösen, die mit diesem Kunden erzielt werden, den kundenspezifischen variablen Kosten gegenüberstellen. Auf einer Schlüsselung fixer Kosten übergeordneter Stufen (Segmentfixkosten, Unternehmensfixkosten) wird dabei verzichtet. Wird jedoch das gesamte Marktsegment ,private Haushalte“ betrachtet, sind neben dessen Erlösen und marktsegmentspezifischen Kosten auch die Kosten der untergeordneten Hierarchiestufen zu berücksichtigen.

Allerdings ist die Umsetzung der Deckungsbeitragsrechnung in der vorgenannten Form hier aus zwei Gründen problematisch. Erstens liegen keine Kostenangaben, sondern lediglich Zahlungsgrößen vor. Die variablen Kosten der Planperiode auf die Zahlungsmittelabflüsse zu reduzieren, führt gegebenenfalls zu einem falschen, möglicherweise zu niedrigen Ansatz variabler Kosten. Dabei ist auch anzumerken, dass aufgrund der Faktenlage eine Deckungsbeitragsmaximierung zum gleichen Ergebnis wie 


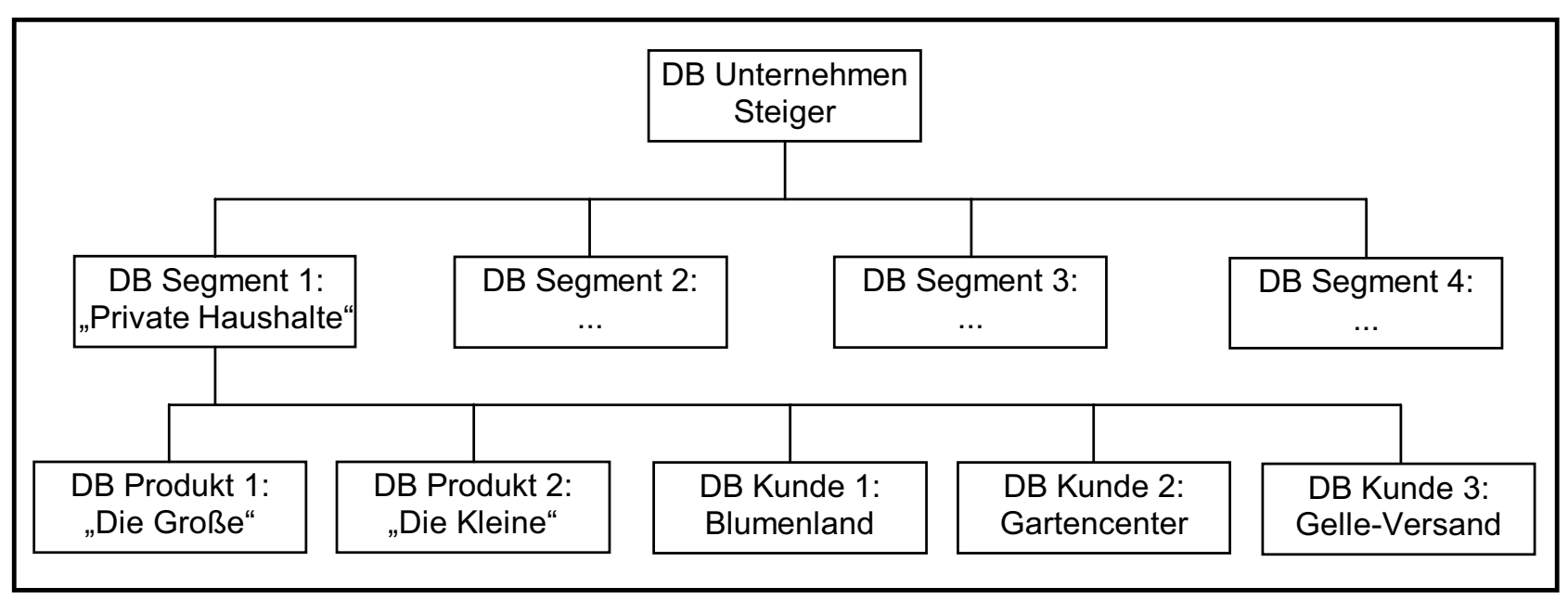

Abb. 1: Mehrstufige Deckungsbeitragshierarchie

\begin{tabular}{|c|c|c|c|}
\hline Unternehmensbereich & Bezeichnung & Variable & Dimension \\
\hline \multirow{5}{*}{ Einkauf } & Rohlinge von Waldmann & RW & [Stück] \\
\hline & Rohlinge von Holzer & $\mathrm{RH}$ & [Stück] \\
\hline & Sprossen von Waldmann & SW & [Stück] \\
\hline & Sprossen von Lindenbaum & $\mathrm{SL}$ & [Stück] \\
\hline & Leim von ABFS & $\mathrm{L}$ & [Liter] \\
\hline \multirow{2}{*}{ Produktion } & „Die Große“ & G & [Stück] \\
\hline & „Die Kleine“ & $\mathrm{K}$ & [Stück] \\
\hline \multirow{4}{*}{ Verkauf } & „Die Große" an Blumenland & GB & [Stück] \\
\hline & "Die Große" an Gartencenter & GG & [Stück] \\
\hline & „Die Kleine“ an Gartencenter & KG & [Stück] \\
\hline & "Die Kleine" an Gelle-Versand & KV & [Stück] \\
\hline
\end{tabular}

Tab. 3: Variablendefinition

eine Cash-Flow-Maximierung führt. Durch die Beschränkung auf Zahlungsgrößen wird somit das Abgrenzungsproblem zwischen Auszahlungen und Kosten ausgeblendet.

Zweitens führt die Preisdifferenzierung im Einkauf und Verkauf dazu, dass keine Stückdeckungsbeiträge für Produkte und/oder Kunden ermittelt werden können. Beispielsweise gelten für verschiedene Kunden unterschiedliche Listenpreise der „Großen“. Folglich kann es für dieses Produkt keinen einheitlichen Stückdeckungsbeitrag geben. Die niedrigstmögliche, aber für die Optimalplanung völlig ausreichende Hierarchieebene, die betrachtet werden kann, ist daher der Segmentdeckungsbeitrag. Eine Analyse anderer Hierarchieebenen ist hier nicht möglich.

Die in der Theorie beliebte Planung mit konstanten Stückdeckungsbeiträgen unterstellt, dass für jedes abgesetzte Produkt der gleiche Preis bezahlt wird und dass diesem einheitliche Kosten der Herstellung zurechenbar sind. Diese Voraussetzung ist jedoch aus praktischer Sicht sehr häufig eine Fiktion. Stattdessen ist in der Regel eine Preisdifferenzierung nach Kundengruppen oder Absatzmärkten anzutreffen. Hinzu treten für verschiedene Kunden individuell vereinbarte Rabatte, Boni oder Skonti. In all diesen Fällen muss für Zwecke der Optimalplanung auf entspre- chend höhere Hierarchieebenen (wie hier der Segmentdeckungsbeitrag) ausgewichen werden.

\section{Ansatz der gemischt ganzzahligen Programmierung}

Sie wollen die Planung mit Hilfe der gemischt ganzzahligen Programmierung (vgl. dazu z. B. Hillier/Lieberman, 1997, S. 376 ff.) durchführen und anschließend die Ergebnisse der Auszahlungsminimierung und der Deckungsbeitragsmaximierung miteinander vergleichen. Der gemischt ganzzahlige Ansatz unterscheidet sich von einem Ansatz der linearen Programmierung lediglich durch die in diesem Fall notwendige Forderung der Ganzzahligkeit einiger Variablen. Zur Formulierung des Ansatzes muss das reale Planungsproblem in ein formales Problem überführt werden. Zunächst bedarf es dazu der Definition von Mengenvariablen in den Bereichen Einkauf, Produktion und Verkauf. Tab. 3 zeigt die Definition sämtlicher Variablen. Alternativen mit weniger Variablen sind dabei denkbar, erzeugen aber nicht zwingend übersichtlichere Ansätze.

Den Vorgaben von Herrn Steiger folgend soll die Lösung des formalen Problems die Mengen angeben, bei denen die 


\begin{tabular}{|c|c|c|c|c|c|}
\hline Unternehmensbereich & Artikel & Lieferant/Kunde & Mindestmenge & Höchstmenge & Nebenbedingungen \\
\hline \multirow{4}{*}{ Einkauf } & Rohlinge & Waldmann & & $10.000[$ Stück] & $R W \leq 10.000$ \\
\hline & Rohlinge & Holzer & & 7.000 [Stück] & $\mathrm{RH} \leq 7.000$ \\
\hline & Sprossen & Waldmann & 5.000 [Stück] & $10.000[$ Stück] & $S W \geq 5.000, S W \leq 10.000$ \\
\hline & Sprossen & Lindenbaum & 5.000 [Stück] & 20.000 [Stück] & $\mathrm{SL} \geq 5.000, \mathrm{SL} \leq 20.000$ \\
\hline \multirow{4}{*}{ Verkauf } & "Die Große“ & Blumenland & & 800 [Stück] & $\mathrm{GB} \leq 800$ \\
\hline & "Die Große“ & Gartencenter & & 500 [Stück] & $\mathrm{GG} \leq 500$ \\
\hline & „Die Kleine“ & Gartencenter & 1.000 [Stück] & 2.000 [Stück] & $K G \geq 1.000, K L \leq 2.000$ \\
\hline & „Die Kleine“ & Gelle-Versand & & $1.500[$ Stück $]$ & $\mathrm{KV} \leq 1.500$ \\
\hline
\end{tabular}

Tab. 4: Einkaufs- und Verkaufsgrenzen

\begin{tabular}{|c|c|}
\hline Forderung & Nebenbedingungen \\
\hline Bedarf Sprossen = Angebot Sprossen & $14 \mathrm{G}+10 \mathrm{~K}=\mathrm{SW}+\mathrm{SL}$ \\
\hline Bedarf Rohlinge = Angebot Rohlinge & $8 \mathrm{G}+6 \mathrm{~K}=\mathrm{RW}+\mathrm{RH}$ \\
\hline Bedarf Leim = Angebot Leim & $((14+6) \mathrm{G}+(10+4) \mathrm{K}) 0,02=\mathrm{L}$ \\
\hline Bedarf „Die Große“ = Angebot „Die Große & $\mathrm{GB}+\mathrm{GG}=\mathrm{G}$ \\
\hline Bedarf „Die Kleine“ = Angebot „Die Kleine“ & $\mathrm{KG}+\mathrm{KV}=\mathrm{K}$ \\
\hline
\end{tabular}

Tab. 5: Mengenkontinuitäten

Summe der Auszahlungen minimiert wird. Auszahlungen entstehen sowohl im Einkauf als auch für die Montage. Die Zielfunktion kann damit wie folgt formuliert werden:

$0,4 \mathrm{RW}+0,35 \mathrm{RH}+0,13 \mathrm{SW}+0,15 \mathrm{SL}+2,5 \mathrm{~L}+$ $(102 \mathrm{G}+70 \mathrm{~K}) 250 / 3600 \rightarrow \min$ !

Die ersten fünf Summanden beschreiben die durch den Einkauf verursachten Auszahlungen. Beispielsweise werden durch den Verbrauch von einem Liter Leim 2,5 € auszahlungswirksam. Der letzte Summand beschreibt dagegen die durch die Montage verursachten Auszahlungen. Die Montage der „Großen“ nimmt dabei 102 Sekunden (6 Holmleimungen (zur Montage von 8 Rohlingen) à $10 \mathrm{Se}-$ kunden +14 Sprossen à 3 Sekunden), die Montage der „Kleinen“ hingegen lediglich 70 Sekunden (4 Holmleimungen à 10 Sekunden +10 Sprossen à 3 Sekunden) in Anspruch. Diese werden anschließend mit dem Auszahlungssatz pro Sekunde ( $250 €$ / 3600 Sekunden) multipliziert. Weitere Auszahlungen fallen nicht an.

Zur Vervollständigung des Ansatzes ist die Berücksichtigung diverser Nebenbedingungen erforderlich. An erster Stelle ist die Deckungsbeitragsrestriktion zu nennen. Bei dieser werden von den Einzahlungen aus dem Verkauf die Auszahlungen, also die Zielfunktion abgezogen:

$135 \mathrm{~GB}+140 \mathrm{GG}+97 \mathrm{KG}+95 \mathrm{KV}-(0,4 \mathrm{RW}+$

$0,35 \mathrm{RH}+0,13 \mathrm{SW}+0,15 \mathrm{SL}+2,5 \mathrm{~L}+$

$(102 \mathrm{G}+70 \mathrm{~K}) 250 / 3600) \geq 150.000$

Bei der Formulierung dieser Nebenbedingung müssen Sie die Skontierung der Absatzprodukte beachten. Daher werden beispielsweise für „die Große“ an Blumenland anstatt $139,18 €$ lediglich $135 €$ einzahlungswirksam. Folgen Sie unterdessen der alternativen Zielgröße, also einer Deckungsbeitragsmaximierung, stellt der Deckungsbeitrag keine Nebenbedingung sondern die Zielfunktion dar, die es zu maximieren gilt.
Neben der Deckungsbeitragsrestriktion gehen die Einkaufs- und Verkaufsgrenzen als eine Gruppe von Nebenbedingungen in die Programmplanung ein. Diese sind in Tab. 4 aufgeführt.

Jede der vorgegebenen Mindest- und Höchstmengen im Einkauf und Verkauf geht als separate Nebenbedingung in den Ansatz ein. So ist beispielsweise für die Variable Sprossen von Lindenbaum sowohl die Mindestabnahmemenge von 5.000 Stück ( $S L \geq 5.000$ ) als auch die Höchstabnahmemenge von 20.000 Stück $(S L \leq 20.000)$ in der Planung zu berücksichtigen.

Ein weiteres wichtiges Element der Modellbildung sind die Mengenkontinuitäten. Sie stellen sicher, dass der vorhandene Bedarf dem Angebot entspricht. Diese Gruppe von Nebenbedingungen ist in Tab. 5 aufgeführt.

Beispielsweise soll die für die Produktion benötigte Sprossenmenge (14 Stück für jede „Große“ und 10 Stück für jede „Kleine“) genau der von den Lieferanten bezogenen Sprossenanzahl entsprechen. Neben den Mengenkontinuitäten muss in der Produktion aber auch eine Montagezeitrestriktion eingehalten werden. Das Angebot der Montagesekunden (1.500 Stunden pro Jahr / 4 . 3.600 Sekunden) ist fix vorgegeben, Leerzeiten sind aber möglich, so dass bei dieser Nebenbedingung anstatt der Identität lediglich die weniger restriktive , $\leq$ “-Bedingung zu fordern ist:

$102 \mathrm{G}+70 \mathrm{~K} \leq 1.350 .000$

Schließlich müssen Sie zwei weitere Gruppen von Nebenbedingungen in der Programmplanung berücksichtigen. Zum einen ist die Nichtnegativität sämtlicher Variablen vorauszusetzen, zum anderen muss - abgesehen von der Variable Leim - auch die Ganzzahligkeit der Variablen sichergestellt werden. 


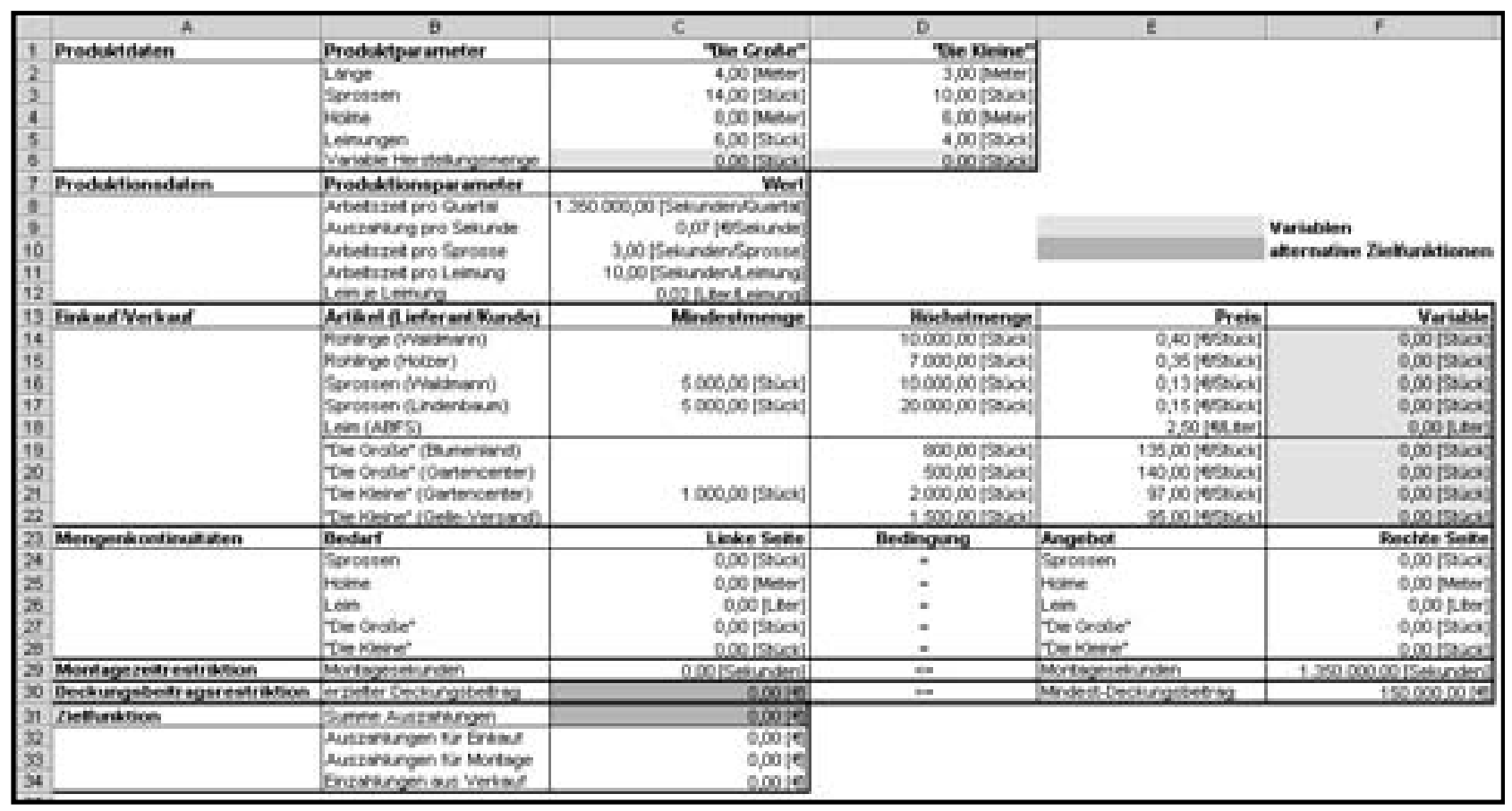

Abb. 2: Excel-Modell

\section{Lösung mit dem Excel-Solver}

Der Excel-Solver, ein Excel Add-In zur Lösung von linearen und nichtlinearen Optimierungsproblemen (vgl. zur Anwendung des Excel-Solvers auf betriebswirtschaftliche Planungsprobleme insbesondere Berens/Delfmann/Schmitting, 2004, S. 233 ff.), ermöglicht Ihnen die Lösung des Planungsproblems. Beim Einsatz des Solvers bedarf es im Anschluss an die Problemanalyse und -definition (vgl. Abschnitt 2 bis 4) eines dreistufigen Vorgehens: Zunächst erfolgt die Abbildung des Problems als Excel-Modell, dann die Definition des zugehörigen Solver-Modells und schließlich die Lösung des Problems mit dem Solver sowie die Interpretation der Ergebnisse.

Im ersten Schritt wird das Problem als Excel-Modell abgebildet. Dazu werden neben sämtlichen Inputdaten die Zielfunktion und die Nebenbedingungen in einem ExcelTabellenblatt aufgelistet und ihre Wirkungszusammenhänge durch entsprechende Formeln zum Ausdruck gebracht. Abb. 2 zeigt eine mögliche Abbildung des Steiger-Modells (das Modell steht unter http://www.wiwi.uni-muenster.de/\%7E20/download/steiger.xls zum Download zur Verfügung).

Die Zeilen 1 bis 12 beinhalten die Inputdaten. Zum einen finden sich hier die Daten zu den Produkten (Zeile 1 bis 6), zum anderen werden die Produktionsdaten aufgeführt (Zeile 7 bis 12). In den Zeilen 13 bis 30 folgen die Nebenbedingungen, zunächst für den Einkauf (Zeile 13 bis 18), dann für den Verkauf (Zeile 19 bis 22) und schließlich die Mengenkontinuitäten sowie die Montagezeit- und Deckungsbeitragsrestriktion (Zeile 23 bis 30). Sowohl die Nichtnegativität als auch die Ganzzahligkeit der Variablen werden im ersten Schritt nicht modelliert, sondern erst bei der Definition des Solver-Modells vorgegeben. Wenn den
Vorgaben von Herrn Steiger, also einer Minimierung der Auszahlungen, gefolgt wird, ist die Zielfunktion des Modells in Zelle C31 einzugeben. Wird hingegen eine Deckungsbeitragsmaximierung angestrebt, stellt Zelle C30 die Zielfunktion dar.

Der zweite Schritt beinhaltet die Definition des zugehörigen Solver-Modells. Dazu rufen Sie den Solver im ExcelMenüpunkt „Extras“ auf. Ist dies nicht möglich, muss der Solver gegebenenfalls unter „Extras/Add-Ins“ aktiviert bzw. installiert werden. Der linke Teil der Abb. 3 zeigt das dann erscheinende Fenster.

Im Eingabefeld „Zielzelle“ wird die Zelle der Zielfunktion aufgenommen. Sie betrachten zunächst die Vorgaben von Herrn Steiger, also eine Minimierung der Auszahlungen und wählen die Zelle C31. Zudem ist festzulegen, ob der Zielwert zu maximieren bzw. zu minimieren ist oder einen bestimmten Wert erreichen soll. Anschließend sind sämtliche Mengenvariablen (Herstellungs- sowie Einkaufs- und Verkaufsmengen) unter den veränderbaren Zellen einzutragen. Nicht zusammenhängende Zellbereiche sind dabei durch ein Semikolon zu trennen. Im unteren linken Eingabefeld werden die Nebenbedingungen eingefügt. Beispielsweise wird durch die erste dort angeführte Bedingung sichergestellt, dass der Deckungsbeitrag mindestens $150.000 €$ beträgt. Die Nebenbedingungen für Einkauf und Verkauf können entweder separat eingegeben oder wie hier blockweise erfasst werden (vgl. die zweite bis vierte Nebenbedingung). Die Bedingung der Ganzzahligkeit wird unterdessen bereits durch die Vorgabe der Ganzzahligkeit der Verkaufsmengen erfüllt (vgl. die fünfte Nebenbedingung), da aufgrund der ganzzahligen Produktionskoeffizienten damit automatisch die Ganzzahligkeit aller Mengenvariablen (abgesehen vom Leim) erzwungen wird. Unter „Optionen“ (vgl. den rech- 


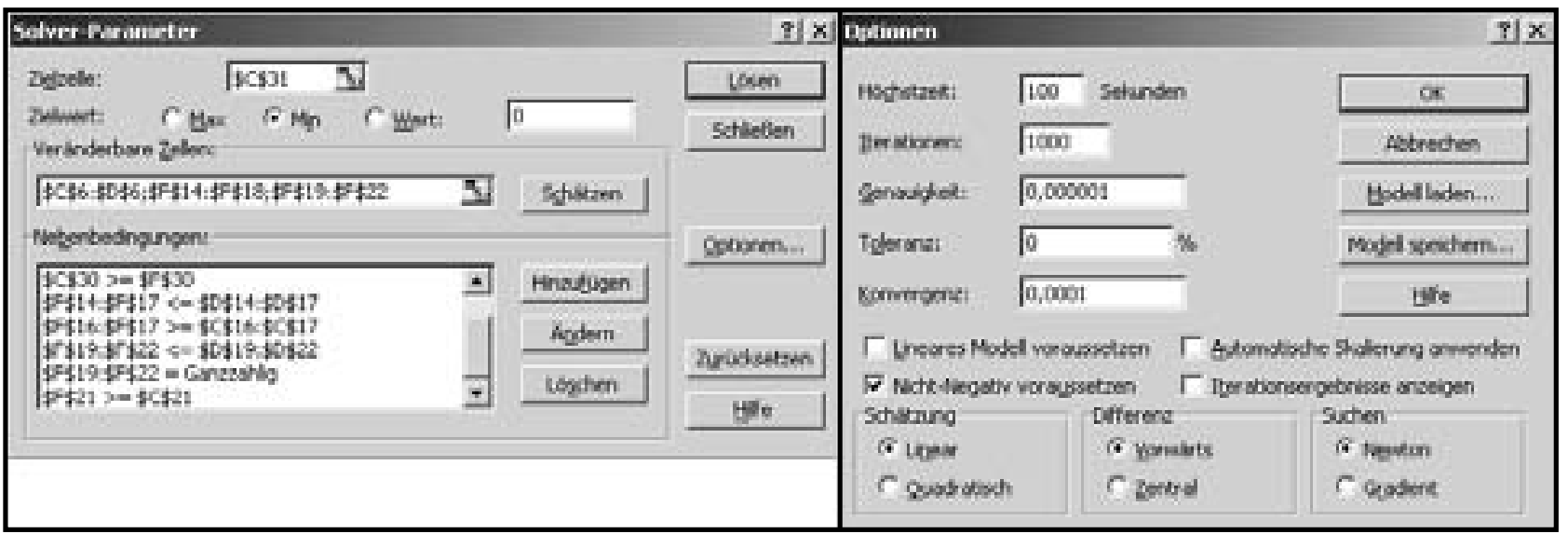

Abb. 3: Solver-Modell

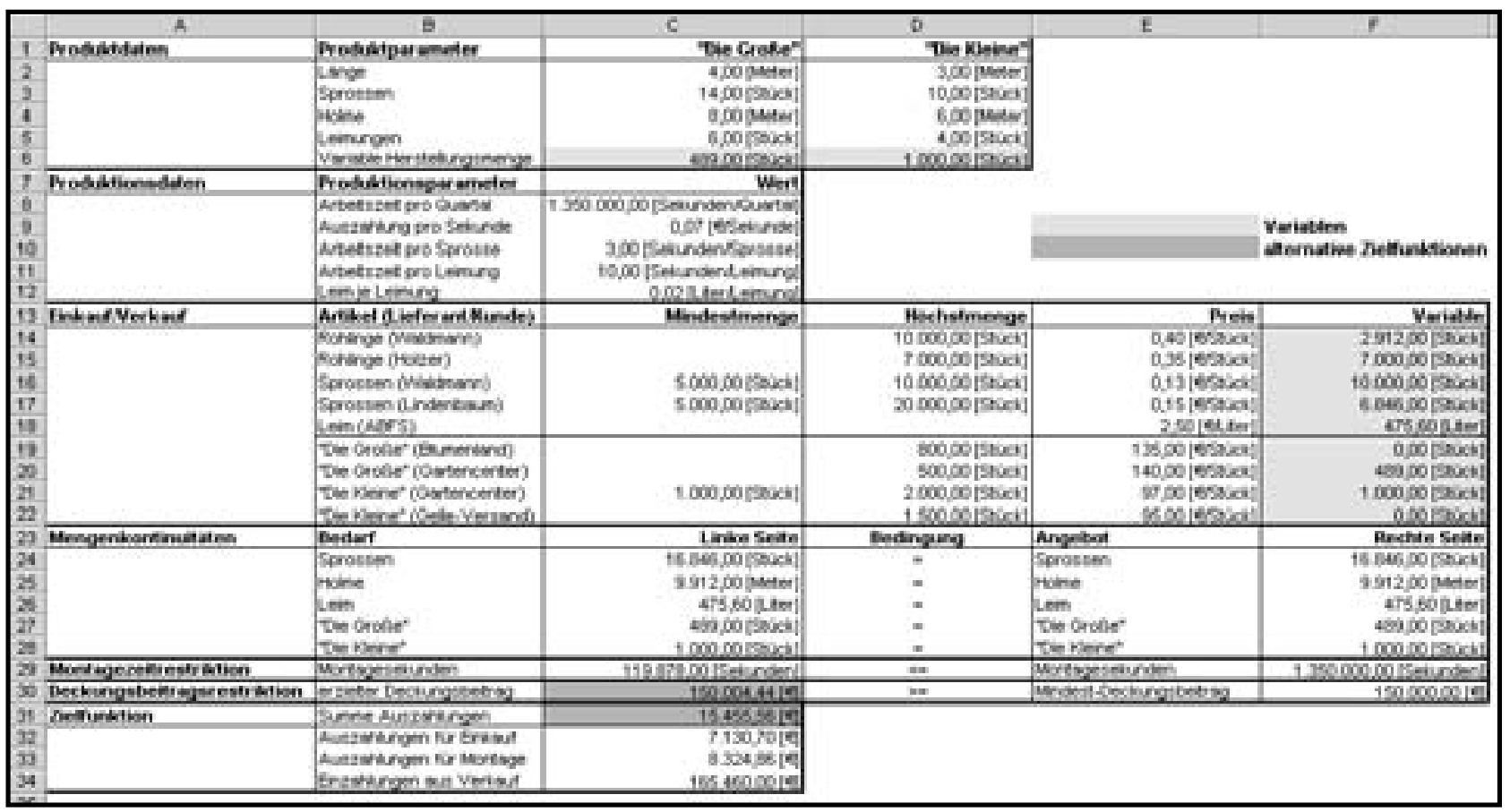

Abb. 4: Ergebnisse

ten Teil der $A b b .3)$ ist zudem die Nichtnegativität der Variablen vorauszusetzen.

Durch das Aufrufen des Buttons „Lösen“ können Sie den Solver starten und das Optimierungsproblem wird gelöst. Es erscheint ein Ergebnismenü, dass Sie durch Aufrufen des Buttons „OK“ bestätigen. Abb. 4 zeigt das dann durch den Solver modifizierte Excel-Tabellenblatt aus Abb. 2.

In dem Tabellenblatt sind die vom Solver ermittelten Werte der Variablen und der Zielfunktion enthalten. Dem Optimierungsergebnis zufolge sind im vorliegenden Fall 489 Mengeneinheiten der „Großen“ (Zelle C6) und 1000 Mengeneinheiten der „Kleinen“ (Zelle D6) zu produzieren. Die minimale Summe der Auszahlungen beträgt dann $15.455,56 €$ (Zelle C31). Es wird ein Deckungsbeitrag von $150.004,44 €$ (Zelle C30) erzielt. Innerhalb der Nebenbedingungen fällt auf, dass mit 119.878 Sekunden (Zelle C29) lediglich 8,88\% des Angebots an Montagezeit von
1.350.000 Sekunden (Zelle F29) verbraucht wird. Diese Restriktion kann selbst bei Ausschöpfung der Absatzhöchstmengen keinen Engpass darstellen und ist daher klar dominiert. Hinsichtlich der weitergehenden Auswertung der Optimierungsergebnisse ist anzumerken, dass die ganzzahlige Programmierung im Gegensatz zur linearen Programmierung keine Sensitivitätsanalysen erlaubt.

Abschließend wollen Sie dieses Optimierungsergebnis mit dem Ergebnis einer Deckungsbeitragsmaximierung vergleichen. Dazu ist im vorliegenden Ansatz lediglich die Minimierung der Zielzelle C31 durch die Maximierung der Zelle C30 zu ersetzen. Das Optimierungsergebnis zeigt dann, dass durch Inkaufnahme einer Auszahlung in Höhe von 27.255,26€ ein deutlich höherer Deckungsbeitrag von $257.444,44 €$ erzielt werden kann. Dazu werden 1.300 Mengeneinheiten der „Großen“ und 1.100 Mengeneinheiten der „Kleinen“ produziert. 
Der Vergleich der Optimierungsergebnisse veranschaulicht sehr deutlich die Vorteilhaftigkeit der Deckungsbeitragsmaximierung gegenüber der Auszahlungsminimierung und damit auch den Widersinn der Zielvorgaben von Herrn Steiger. Schließlich entgeht der Steiger GmbH durch die Vorgabe der Auszahlungsminimierung ein zusätzlicher Deckungsbeitrag in Höhe von 107.440,41 €, der zur Sicherung der wirtschaftlichen Zukunft des Unternehmens dringend nötig wäre. Mit den Optimierungsergebnissen in der Hand klopfen Sie daher an die Bürotür von Herrn Steiger. $\cdots$

\section{Literatur}

Berens, W., W. Delfmann, W. Schmitting, Quantitative Planung, 4. Aufl., Stuttgart 2004.

Coenenberg, A. G., Kostenrechnung und Kostenanalyse, 5. Aufl., Stuttgart 2003.

Hillier, F. S., G. S. Lieberman, Operations Research. Einführung, 5. Aufl., München, Wien 1997.
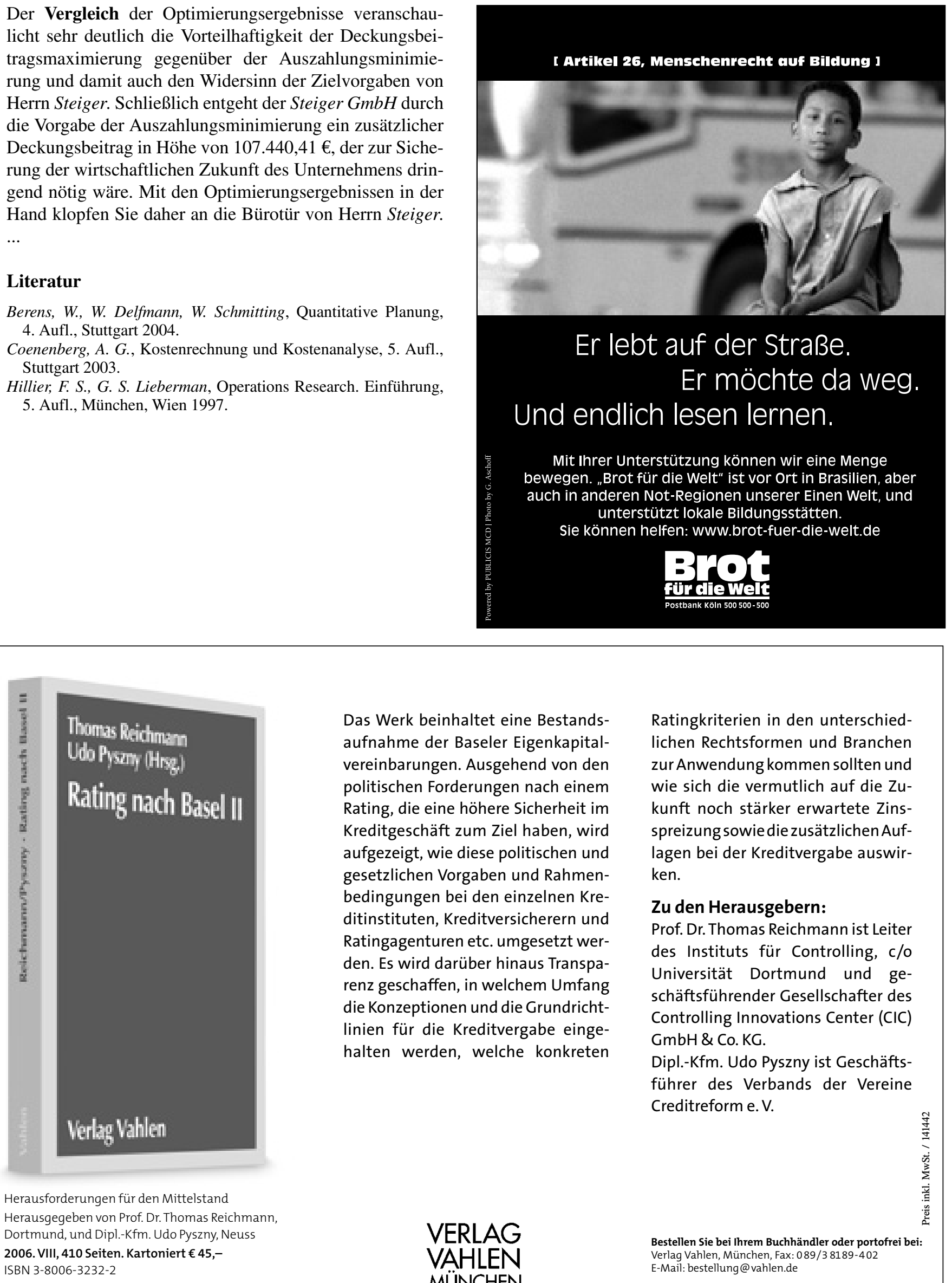

Das Werk beinhaltet eine Bestandsaufnahme der Baseler Eigenkapitalvereinbarungen. Ausgehend von den politischen Forderungen nach einem Rating, die eine höhere Sicherheit im Kreditgeschäft zum Ziel haben, wird aufgezeigt, wie diese politischen und gesetzlichen Vorgaben und Rahmenbedingungen bei den einzelnen Kreditinstituten, Kreditversicherern und Ratingagenturen etc. umgesetzt werden. Es wird darüber hinaus Transparenz geschaffen, in welchem Umfang die Konzeptionen und die Grundrichtlinien für die Kreditvergabe eingehalten werden, welche konkreten
Ratingkriterien in den unterschiedlichen Rechtsformen und Branchen zur Anwendung kommen sollten und wie sich die vermutlich auf die $\mathrm{Zu}$ kunft noch stärker erwartete Zinsspreizung sowie die zusätzlichen Auflagen bei der Kreditvergabe auswirken.

\section{Zu den Herausgebern:}

Prof. Dr. Thomas Reichmann ist Leiter des Instituts für Controlling, c/o Universität Dortmund und geschäftsführender Gesellschafter des Controlling Innovations Center (CIC) $\mathrm{GmbH} \&$ Co. KG

Dipl.-Kfm. Udo Pyszny ist Geschäftsführer des Verbands der Vereine Creditreform e. V. 